\title{
Medida da Participação e do Ambiente - Crianças Pequenas (YC-PEM): tradução e adaptação transcultural para o uso no Brasil
}

\section{Young Children's Participation and Environment Measure (YC-PEM): translate and cross-cultural adaptation for use in Brazil}

\author{
João Antonio da Silva Filho ${ }^{1}$, Ana Paula Martins Cazeiro ${ }^{2}$, Ana Carolina de \\ Campos $^{3}$, Egmar Longo ${ }^{4}$
}

http://dx.doi.org/10.11606/issn.2238-6149.v30i3p140-149

\begin{abstract}
Silva Filho JA, Cazeiro APM, Campos AC, Longo E. Medida da Participação e do Ambiente - Crianças Pequenas (YC-PEM): tradução e adaptação transcultural para o uso no Brasil. Rev Ter Ocup Univ São Paulo. 2020 set.-dez.;30(3):140-9.
\end{abstract}

RESUMO: Introdução: A Medida da Participação e do Ambiente Crianças Pequenas (YC-PEM) é uma ferramenta que avalia a frequência, envolvimento e impacto do ambiente na participação de crianças entre zero e cinco anos de idade, com e sem deficiência, nos ambientes da casa, creche/pré-escola e comunidade. Objetivo: Traduzir e adaptar culturalmente a YC-PEM para o uso no Brasil. Método: O processo foi composto por seis etapas - (1) Tradução do inglês-Norte americano para o português-Brasil; (2) Síntese das traduções; (3) Análise por comitê de especialistas; (4) Entrevista cognitiva com os pais/responsáveis; (5) Retro tradução; (6) Consolidação da versão final. Resultados: Concomitantemente à tradução, foram necessárias adaptações para facilitar a leitura e interpretação da ferramenta. Houve alteração nas instruções para preenchimento. Alguns itens referentes à participação e ambiente foram alterados e receberam outros exemplos mais adequados ao contexto brasileiro. Conclusão: O processo de tradução e adaptação transcultural da YC-PEM Brasil seguiu uma metodologia padronizada e se considera que o resultado foi satisfatório, sendo necessária a condução de pesquisas adicionais para a sua validação neste país.

DESCRITORES: Criança; Participação social; Inquéritos e questionários; Classificação Internacional de Funcionalidade, Incapacidade e Saúde.
Silva Filho JA, Cazeiro APM, Campos AC, Longo E. Young Children's Participation and Environment Measure (YC-PEM): translate and cross-cultural adaptation for use in Brazil. Rev Ter Ocup Univ São Paulo. 2020 Sept.-Dec.;30(3):140-9

\begin{abstract}
Introduction: The Young Children's Participation and Environment Measure (YC-PEM) is a tool used to evaluate the frequency, involvement and impact of the environment on participation of children with and without disabilities between zero and five years of age in the home, daycare/preschool and community contexts. Objective: To translate and culturally adapt YC-PEM for use in Brazil. Method: The process consisted of six steps - (1) Translation from English-North American to PortugueseBrazil; (2) Synthesis of translations; (3) Analysis by an expert committee; (4) Cognitive interviews with parents/guardians; (5) Retro translation; (6) Consolidation of the final version. Results: Concomitantly with the translation, adaptations were necessary to facilitate the reading and interpretation of the tool. The title structure and the instructions for users were changed. Some items related to participation and environment were changed and received other examples more related to the Brazilian context. Conclusion: YC-PEM Brazil can help caregivers and healthcare professionals to identify the level of participation of children between zero and five years of age and the need for interventions aimed at improving their participation in the environment.
\end{abstract}

KEYWORDS: Child; Social participation; Surveys and questionnaires; International Classification of Functioning, Disability and Health.

Parte do mestrado "Young Children's Participation and Environment Measure (YC-PEM): Tradução e adaptação transcultural para o uso no Brasil" de João Antonio da Silva Filho, Programa de Pós-Graduação em Ciências da Reabilitação, FACISA - UFRN. Resultados parciais desta pesquisa foram apresentados durante o estágio de docência e pesquisa na Universidad Autónoma de Manizales, Manizales, Colômbia, jun.-ago. 2018.

1. Programa de Pós-Graduação em Ciências da Reabilitação da Faculdade de Ciências da Saúde do Trairi (FACISA). Universidade Federal do Rio Grande do Norte (UFRN), Santa Cruz, RN, BR. ORCID: https://orcid.org/0000-0002-5486-5080. E-mail: joaofilho.pb@hotmail.com.

2. Departamento de Terapia Ocupacional da Universidade Federal do Rio de Janeiro (UFRJ), Rio de Janeiro, RJ; Departamento de

Fundamentos da Educação da Universidade Federal do Ceará (UFC), Fortaleza, CE, BR. ORCID: https://orcid.org/0000-0002-0812-8987.

E-mail: paulacazeiro@gmail.com.

3. Departamento de Fisioterapia da Universidade Federal de São Carlos (UFSCar), São Carlos, SP, BR. ORCID: https://orcid.org/ 0000-0001-7267-4467. E-mail: campos.anacarol@gmail.com.

4. Curso de Fisioterapia e Programas de Pós-Graduação em Ciências da Reabilitação e Saúde Coletiva da Faculdade de Ciências da Saúde do Trairi (FACISA), Universidade Federal do Rio Grande do Norte (UFRN), Santa Cruz, RN, BR. ORCID: https://orcid.org/ 0000-0001-6263-1818. E-mail: egmarlongo@yahoo.es.

Endereço para correspondência: Egmar Longo. Programa de Pós-Graduação em Ciências da Reabilitação, Faculdade de Ciências da Saúde do Trairi (FACISA), Universidade Federal do Rio Grande do Norte (UFRN). Rua Vila Trairi - Santa Cruz, RN, BR. CEP: 59200-000. E-mail: egmarlongo@yahoo.es. 


\section{INTRODUÇÃO}

$\mathrm{D}^{\mathrm{s}}$ esde a publicação da Classificação Internacional de Funcionalidade, Incapacidade e Saúde (CIF) pela Organização Mundial da Saúde (OMS) ${ }^{1}$ em 2001, o interesse pela participação tem crescido exponencialmente, especialmente no campo da reabilitação infantil ${ }^{2}$. Participação é o resultado de saúde e bem-estar e, em crianças pequenas, está vinculado a resultados de desenvolvimento ${ }^{3,4}$. Crianças com deficiência participam menos frequentemente em atividades em casa e na comunidade, ${ }^{5}$ tendo sido descrita a existência de barreiras físicas, sociais e atitudinais ${ }^{6,7,8,9}$.

Há evidências de que o ambiente impacta na participação de crianças com deficiências e que intervenções focadas nos fatores ambientais melhoram os níveis de envolvimento delas em casa, na escola e na comunidade ${ }^{5,10}$. Para efetivar intervenções desta natureza, é preciso conhecer os aspectos que podem agravar os níveis de incapacidade e dependência (barreiras), bem como aqueles que podem aumentar os níveis de participação (facilitadores).

Alguns instrumentos estão validados para uso no Brasil, a exemplo do CHORES (Children Helping Out: Responsibilities, Expectations and Supports) ${ }^{11}$, que avalia a participação das crianças e adolescentes no ambiente doméstico, e do CHIEF (Craig Hospital Inventory of Environmental Factors) ${ }^{12}$, que tem por função avaliar as barreiras e facilitadores do ambiente que influenciam na participação de crianças e adolescentes. Porém, nenhuma ferramenta avalia simultaneamente a participação, o ambiente, as barreiras e os facilitadores da participação de crianças pequenas, especialmente para uso no contexto cultural brasileiro.

A "Young Children's Participation and Environment Measure" (YC-PEM), denominada Medida da Participação e do Ambiente - Crianças Pequenas, disponibilizada em 2013 pelo Centre for Childhood Disability Research - CanChild (https://www.canchild.ca/), é uma ferramenta baseada na CIF que considera a percepção de pais/responsáveis acerca da participação e do ambiente de crianças pequenas. Avalia a frequência e o grau de envolvimento nas atividades realizadas em casa, na creche/pré-escola e na comunidade, assim como o impacto do ambiente na participação da criança em cada um destes três contextos ${ }^{13}$.

A YC-PEM pode beneficiar pais, prestadores de serviços e pesquisadores ao permitir a identificação das atividades em que a criança não participa e deseja ou necessita participar, bem como os fatores que facilitam ou dificultam tal participação, possibilitando a incorporação de medidas favoráveis ao cotidiano da criança nos ambientes domiciliar, escolar e comunitário ${ }^{14,15}$. Desta forma, o objetivo deste estudo foi traduzir e adaptar culturalmente a Young Children's Participation and Environment Measure (YC-PEM) para uso no Brasil.

\section{MÉTODO}

Trata-se de um estudo metodológico que envolveu a tradução e adaptação transcultural da Young Children's Participation and Enviroment Measure (YC-PEM) para uso no Brasil. Participaram 13 sujeitos, residentes nas cidades de Guarabira - PB, Macaíba - RN, Lagoa Salgada - RN, São Gonçalo - RN e São Carlos - SP, recrutados por conveniência, durante o período de julho a agosto de 2019. Os critérios de inclusão foram: pais ou responsáveis de crianças com idades entre zero e cinco anos, com e sem deficiência, de ambos os sexos, mediante aceitação para participar na pesquisa através da assinatura do Termo de Consentimento Livre e Esclarecido (TCLE) e Termo de Autorização para Gravação de Voz. Seriam excluídos do estudo a qualquer momento todos os sujeitos que desejassem retirar seu consentimento de participação.

\section{Young Children's Participation and Enviroment Measure (YC-PEM)}

A YC-PEM é uma ferramenta que visa avaliar a percepção de pais ou responsáveis por crianças entre zero e cinco anos de idade, com e sem deficiências ou atraso do desenvolvimento, acerca do seu nível de participação em diferentes atividades e os fatores ambientais que possam favorecer ou dificultar tal participação. Esta ferramenta segue o modelo da PEM-CY (Participation and Environment Measure for Children and Youth), já adaptada para o Brasi ${ }^{16}$, e combina a avaliação da participação e do ambiente em um único instrumento ${ }^{13}$.

No ambiente domiciliar, a YC-PEM descreve a participação das crianças em 13 tipos de atividades, sendo eles: descansar; cuidados pessoais; limpar-se; alimentação; organização; preparar refeições; cuidar de outros membros da família; lavar roupa ou louça; artes, artesanato, histórias, música; hora dos eletrônicos; jogos e brincadeiras em casa; comemorações em casa; visitas em casa. No ambiente da creche/pré-escola, são avaliados três tipos de atividades (atividades em grupo; socialização com amigos; passeios e eventos), enquanto que no ambiente da comunidade são avaliados 11 tipos: compras ou serviços; comer fora; compromissos; aulas e cursos; atividades físicas organizadas; atrações ou locais da comunidade; encontros e atividades religiosas; 
encontros sociais; eventos comunitários; atividades físicas livres; viagens, férias e passeios fora da comunidade. Em cada item, o respondente é solicitado a pensar em todas as atividades daquele tipo que se aplicam a sua criança e a responder a três perguntas, que dizem respeito à frequência de participação (escala de oito pontos que varia entre "nunca" e "uma ou mais vezes por dia"), grau de envolvimento da criança (escala de três pontos - pouco; mais ou menos; muito envolvida) e desejo de mudança na participação da criança (não ou sim, sendo este com cinco opções para o tipo de mudança desejada).

Para cada ambiente, os pais/responsáveis podem selecionar os aspectos que facilitam ou tornam mais difícil a participação das crianças, tais como a disposição dos móveis, os estímulos sensoriais, as atitudes das pessoas e as habilidades motoras, cognitivas e sociais exigidas para a realização das atividades (quatro opções de resposta - não faz diferença; geralmente ajuda; às vezes ajuda/às vezes dificulta; geralmente torna mais difícil). Também são avaliados os recursos disponíveis ou adequados (por exemplo, materiais, transporte, dinheiro, tempo) para ajudar na participação da criança (quatro opções: não é necessário; geralmente sim; às vezes sim/às vezes não; geralmente não).

\section{Procedimentos}

O Canchild, que detém os direitos de venda e divulgação da YC-PEM, autorizou a sua tradução pelo grupo de pesquisa. Este estudo foi desenvolvido de acordo com a Resolução $n^{\circ}$. 466/12, do Conselho Nacional de Saúde (CNS) - Comissão Nacional de Ética em Pesquisa (CONEP), tendo sido avaliado e aprovado pelo Comitê de Ética em Pesquisa da Faculdade de Ciências da Saúde do Trairi (FACISA) da Universidade Federal do Rio Grande do Norte (UFRN), número de parecer 2.388.525/2017 e CAAE 79628017.0.0000.5568. Os objetivos da investigação foram explicados aos participantes, que assinaram o Termo de Consentimento Livre e Esclarecido (TCLE) concordando com os procedimentos.

Trata-se de um estudo metodológico conduzido por pesquisadores de quatro Instituições brasileiras (UFRN, UFRJ, UFC e UFSCar), sendo que o processo de tradução e adaptação transcultural da YC-PEM seguiu uma metodologia padronizada ${ }^{17}$ em seis etapas:

(1) Tradução inicial - A YC-PEM original foi traduzida do inglês norte americano para o português do Brasil, independentemente, por duas pesquisadoras bilíngues, docentes universitárias e especialistas na área de reabilitação, cuja língua nativa é o português brasileiro.
(2) Síntese das traduções - Após as traduções iniciais independentes, foi produzida a versão consensual entre as duas pesquisadoras brasileiras.

(3) Análise pelo comitê de especialistas da versão préfinal - composto por sete terapeutas ocupacionais (duas de MG, três do RJ, uma de SP e uma do RS) e uma fisioterapeuta de MG. Destas oito juízas consultadas, cinco são professoras universitárias. Todas avaliaram independentemente a clareza, relevância e adequação dos itens à realidade do Brasil, verificando a adequação conceitual, semântica (vocabulário e gramática) e cultural da versão traduzida (brasileira). Para esta etapa, foi enviado por e-mail um quadro contendo todas as instruções e questões do YC-PEM. Cada item do instrumento era acompanhado de perguntas acerca do grau de compreensão e pertinência do item para a faixa etária e cultura brasileira (escalas de 0 a 10 pontos), bem como era fornecido espaço para sugestões de modificação.

(4) Entrevista cognitiva com os pais/responsáveis de crianças entre zero e cinco anos de idade - aplicada a um grupo de sete responsáveis por crianças com deficiência e outro grupo composto por seis responsáveis por crianças sem deficiência. Entrevistas cognitivas utilizam um conjunto de componentes sociais e comunicativos cruciais para a coleta de informações, tais como estabelecer uma boa relação com o entrevistado, explicar os objetivos da entrevista, promover um relato livre e então acrescentar questões baseadas no discurso do indivíduo, fazer síntese de alguns pontos centrais do relato, dando a oportunidade do entrevistado acrescentar nova informação ou mesmo fazer autocorreção, e finalizar o processo garantindo que o sujeito saiu do local da entrevista calmo e seguro ${ }^{18}$. Para esta etapa da pesquisa, foi utilizado um roteiro de entrevista cognitiva baseada em Paulo et al. ${ }^{18}$ a partir do qual foram apresentadas questões abertas ao entrevistado após cada seção do YC-PEM, indagando a sua opinião sobre os itens do teste, sugestões de palavras que pudessem melhorar a sua compreensão, a possibilidade de encontrar respostas para todas as perguntas do instrumento, a pertinência das atividades incluídas no instrumento para a faixa etária e para a cultura brasileira e a existência de outras atividades relevantes não incluídas no teste.

(5) Retro tradução - Tradução reversa realizada por uma terceira pesquisadora bilíngue, docente universitária especialista na área da reabilitação, cuja língua nativa é o português brasileiro. Esta pesquisadora não teve acesso à versão original do instrumento.

(6) Consolidação da versão final - A versão retrotraduzida foi encaminhada ao CanChild para aprovação da versão final. Tais etapas estão sintetizadas na Figura 1. 
Silva Filho JA, et al. Medida da Participação e do Ambiente - Crianças Pequenas. Rev Ter Ocup Univ São Paulo. 2020 set./dez:;30(3):140-9.

Processo de tradução e adaptação transcutural da YC-PEM

Versão YC-PEM original

(inglês norte americano)

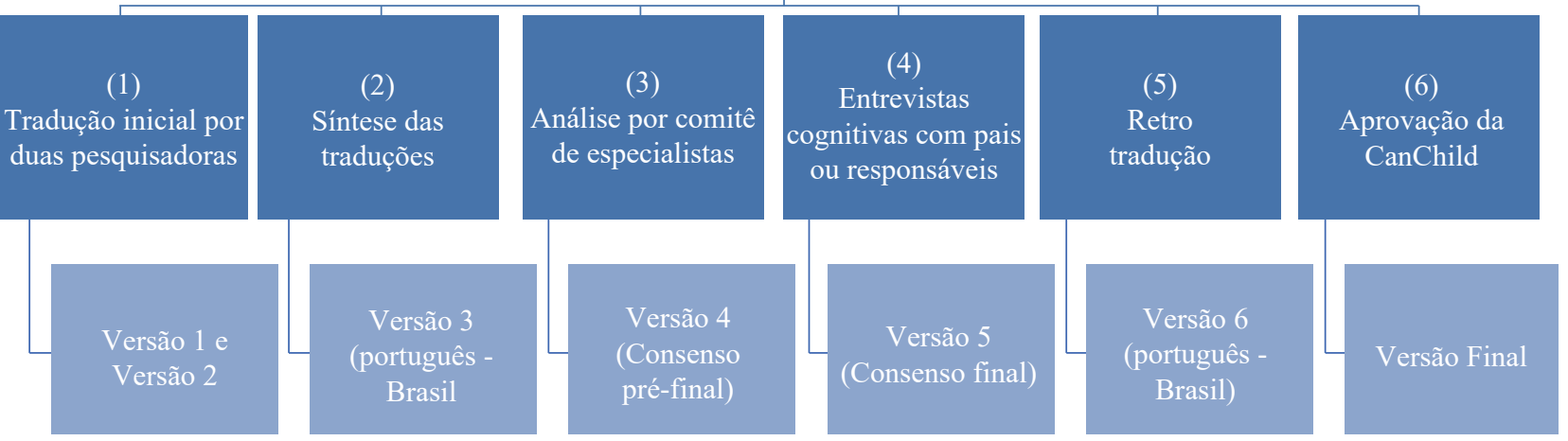

Figura 1 - Resumo metodológico do processo de tradução e adaptação transcultural do Instrumento YC-PEM para uso no Brasil.

\section{RESULTADOS}

Durante a etapa 1 - traduções independentes para o português do Brasil realizadas por duas pesquisadoras alguns termos sofreram adaptações com o propósito de se aproximar da realidade cultural do país (versões 1 e $2 \mathrm{em}$ português brasileiro).

Na etapa 2 - síntese das traduções realizada pelas mesmas pesquisadoras - todas as discrepâncias encontradas no estágio anterior foram cuidadosamente analisadas e os termos considerados mais usuais no Brasil foram escolhidos para a versão 3 (português brasileiro).

Na etapa 3 - análise por comitê de especialistas - as sugestões apresentadas pelas especialistas foram analisadas por quatro pesquisadores, que realizaram as alterações, melhorias e adaptações relevantes dos itens, seus respectivos exemplos e opções de respostas (Tabela 1), de modo a produzir a versão 4 (consenso pré-final).

Tabela 1 - Alterações dos itens após a etapa 3 (Análise do comitê de especialistas) da tradução e adaptação transcultural do YC-PEM

\begin{tabular}{lll}
\hline Seção & YC-PEM traduzido* (Versão 3) & Alteração de item** (Versão 4) \\
\hline $\begin{array}{l}\text { Envolvimento } \\
\text { - Participação }\end{array}$ & $\begin{array}{l}\text { Pense em 1 ou 2 atividades deste tipo, considerando } \\
\text { aquelas em que sua criança participa com mais } \\
\text { frequência. Geralmente, quão } \\
\text { eriança quando? }\end{array}$ & $\begin{array}{l}\text { Pense em 1 ou 2 atividades deste tipo, considerando } \\
\text { aquelas em que sua criança participa com mais frequência. } \\
\text { Geralmente, como é o envolvimento dela quando faz este } \\
\text { tipo de atividade? }\end{array}$ \\
\hline $\begin{array}{l}\text { Gostaria que a participação da sua criança mudasse } \\
\text { neste tipo de rotina? }\end{array}$ & $\begin{array}{l}\text { Gostaria que a participação da sua criança mudasse neste } \\
\text { tipo de atividade de cuidado básico? }\end{array}$ \\
$\begin{array}{l}\text { mudança - } \\
\text { Participação }\end{array}$ & Sim, ser mais interativa & Sim, $\underline{\text { interagir mais }}$ \\
& Sim, ser mais eooperativa & Sim, colaborar mais \\
\hline
\end{tabular}


Tabela 1 - Alterações dos itens após a etapa 3 (Análise do comitê de especialistas) da tradução e adaptação transcultural do YC-PEM

\begin{tabular}{ll}
\hline Seção & YC-PEM traduzido* (Versão 3) \\
\hline & B1. Limpeza (ex. guardar os brinquedos, jogar o \\
& lixo fora, varrer o ehão ou limpar a easa, etridar do jartim, \\
& limparo quintat). \\
& B2. Preparar refeições (ex. guardar as compras, medir e \\
Participação & misturar ingredientes, colocar os pratos na mesa). \\
em casa & B3. Cuidar de outros membros da família (ex. irmãos, \\
& bichos de estimação). \\
& D2. Visitas em casa (ex. receber amigos e familiares, \\
& convidar amigos para brincar, festa do pijama ou dormir \\
& com amigos).
\end{tabular}

3. Os aspectos físicos das atividades de casa

(ex. força muscular, esforęo físieo, coordenação dos movimentos, cansaço).

4. Os aspectos mentais das atividades de casa (ex. concentração, atenção, resolução de problemas).

5. Os aspectos sociais das atividades de casa (ex. comunicação, interação com os outros).

6. O relacionamento da criança com pessoas da família em casa (ex. irmãos, pais ou padrasto/madrasta,

Ambiente da avós, parentes).

casa 8. Normas (ex. regras do condomínio, do local de trabalho dos pais, tieenȩa familiar, trabalhar em casa, folgas, horas de trabalho).

9. Serviços em casa (ex. terapeutas, babás, cuidador profissional, etc).

10. Materiais (ex. brinquedos, comida, móveis, fraldas, roupas, dinheiro, TV, computador, eletricidade, internet). 11. Informação (ex. orientação sobre como realizar as atividades em casa, sobre serviços e programas disponíveis).

Participação

na creche/pré- A3. Passeios e eventos (ex. noite do pijama, ir à biblioteca, escola festa na escola, apresentação de dança/teatro).

1. A maneira como os móveis estão organizados, a limpeza, espaço seguro na sala, rampas, elevadores e escadas do prédio.

3. Condições do tempo nas áreas abertas (ex. temperattra, elima).

9. Políticas e procedimentos da escola (ex. critérios para matrícula, políticas de atendimento, proeedimentos de entrada, relatórios de aeidentes, regras de comportamento). 11. Acesso a transporte público para ir para a creche/préescola (ex. ônibus, trem, metrô, lotação, balsa).

12. Programas e serviços da creche/pré-escola (ex. sala de Ambiente da apoio, monitor de apoio/acompanhante, reetrros especiais). creche/pré- 13. Materiais (ex. adaptações, brinquedos adaptados, escola materiais para artes e artesanato, opções de comida saudável, móveis adequados, acesso à internet e tecnologia para ajudar nas aulas, banheiros aeessíveis, salas de aula e parquinho).

14. Informação (ex. orientação sobre como realizar as atividades escolares, sobre serviços e recursos disponíveis).

16. Você (e sua família) tem dinheiro suficiente para ajudar na participação da criança na creche/pré-escola (ex. pagar taxas-ou mensalidades, comprar material escolar, participar de campanhas de arrecadação de dinheiro ou alimentos)?
Alteração de item** (Versão 4)

Continuação

B1. Limpeza (ex. guardar os brinquedos, usar a lixeira, limpar a mesa, cuidar das plantas, arrumar a cama, organizar suas coisas).

B2. Preparar refeições (ex. guardar as compras, medir e misturar ingredientes, colocar os pratos na mesa, escolher o alimento).

B3. Cuidar de outros membros da família (ex. irmãos, primos, avós, bichos_de estimação).

D2. Visitas em casa (ex. receber amigos e familiares, convidar amigos para brincar, dormir com familiares ou amigos).

3. Os aspectos físicos exigidos para realizar as atividades de casa (ex. força muscular, coordenação dos movimentos, cansaço).***

4. Os aspectos mentais exigidos para realizar as atividades de casa (ex. concentração, atenção, resolução de problemas).***

5. Os aspectos sociais exigidos para realizar as atividades de casa (ex. comunicação, interação com os outros).***

6. O relacionamento da criança com familiares em casa (ex. irmãos, pais ou padrasto/madrasta, avós, parentes).

8. Normas (ex. regras do condomínio, da casa, do local de trabalho dos pais, trabalhar em casa, folgas, horas de trabalho).

9. Serviços em casa (ex. terapeutas, babás, cuidador profissional, agentes de saúde, etc).

10. Recursos e materiais em casa (ex. brinquedos, comida, móveis, fraldas, roupas, TV, computador, eletricidade, internet, etc).

11. Informação (ex. receber orientação sobre como realizar as atividades em casa, sobre serviços disponíveis, direitos, etc).

A3. Passeios e eventos (ex. noite do pijama, ir à

biblioteca, festa na escola, visitas com a escola, apresentação de dança/teatro).

1. A maneira como os móveis estão organizados, a limpeza, a segurança, presença de rampas, elevadores e escadas do prédio. 3. Condições do tempo nas áreas abertas (ex. muito calor, muito frio, muita chuva).

9. Políticas e procedimentos da escola (ex. critérios para matrícula, políticas de atendimento e acompanhamento do aluno, comunicação entre escola e família, regras de comportamento).

11. Acesso a transporte público para ir para a creche/pré-escola (ex. ônibus, trem, metrô, lotação, balsa, transporte escolar).

12. Programas e serviços da creche/pré-escola (ex. sala de apoio, monitor de apoio/acompanhante, auxiliar do professor). 13. Recursos e materiais (ex. adaptações, brinquedos adaptados, materiais para artes, opções de comida saudável, móveis adequados, acesso à internet e tecnologia para ajudar nas aulas, banheiros, salas de aula e parquinho acessíveis).

14. Informação (ex. receber orientação sobre como realizar as atividades escolares, sobre serviços e recursos disponíveis.

16. Você (e sua família) tem dinheiro suficiente para ajudar na participação da criança na creche/pré-escola (ex. pagar taxas, mensalidades ou transportes, comprar material escolar, participar de campanhas de arrecadação de dinheiro ou alimentos)? 
Silva Filho JA, et al. Medida da Participação e do Ambiente - Crianças Pequenas. Rev Ter Ocup Univ São Paulo. 2020 set./dez.;30(3):140-9.

Tabela 1 - Alterações dos itens após a etapa 3 (Análise do comitê de especialistas) da tradução e adaptação transcultural do YC-PEM

\begin{tabular}{|c|c|c|}
\hline Seção & YC-PEM traduzido* (Versão 3) & Alteração de item** (Versão 4) \\
\hline $\begin{array}{l}\text { Participação } \\
\text { na } \\
\text { comunidade }\end{array}$ & $\begin{array}{l}\text { A3. Compromissos (ex. cortar cabelo, ir ao dentista, } \\
\text { consulta médica). } \\
\text { B1. Aulas e cursos (ex. aulas de música, artesanato, curso } \\
\text { de inglês out espanhol). } \\
\text { C1. Atrações ou locais da comunidade (ex. biblioteca } \\
\text { e livraria, museus, cinema, aquário, horta, zoológico, } \\
\text { fazendinha). } \\
\text { D1. Atividades físicas livres (ex. ir a parques e } \\
\text { parquinhos, praias, fazer caminhadas, andar de bicicleta ou } \\
\text { pattins, pescar). }\end{array}$ & $\begin{array}{l}\text { A3. Compromissos (ex. cortar cabelo, ir ao dentista, } \\
\text { consulta médica, terapias). } \\
\text { B1. Aulas e cursos (ex. aulas de música, artesanato, curso } \\
\text { de inglês, espanhol, etc). } \\
\text { C1. Atrações ou locais da comunidade (ex. biblioteca e } \\
\text { livraria, museus, cinema, teatro, aquário, horta, zoológico, } \\
\text { fazendinha). } \\
\text { D1. Atividades físicas livres (ex. ir a parques e } \\
\text { parquinhos, praias, fazer caminhadas, andar de bicicleta } \\
\text { ou patinete, pescar). }\end{array}$ \\
\hline $\begin{array}{l}\text { Ambiente da } \\
\text { comunidade }\end{array}$ & $\begin{array}{l}\text { 8. Condições de tempo nas áreas abertas } \\
\text { (ex. temperattra, elima). } \\
\text { 10. Normas (ex. leis, regras da vizinhanȩa, políticas de } \\
\text { cuidados com as crianças, leis de trabalho para os pais). } \\
\text { 13. Programas e serviços da comunidade (ex. programas de } \\
\text { esporte adaptado, etridador profissional/acompantrante). } \\
\text { 14. Materiais (ex. bolsa de bebê, comida, brinquedos, } \\
\text { fraldas, roupas, carrinho de bebê, adaptações, cadeira de } \\
\text { rodas). } \\
\text { 15. Informação (ex. sobre como realizar as atividades } \\
\text { na eomtnidade, sobre eventos, serviços e recursos } \\
\text { disponíveis). }\end{array}$ & $\begin{array}{l}\text { 8. Condições de tempo nas áreas abertas (ex. muito calor, } \\
\text { muito frio, muita chuva). } \\
\text { 10. Normas (ex. leis, regras da comunidade, políticas de } \\
\text { cuidados com as crianças, regras do trabalho } \underline{\text { dos pais). }} \\
\text { 13. Programas e serviços da comunidade (ex. esporte } \\
\text { adaptado, brinquedoteca, serviços de saúde, } \\
\text { centro comunitário). } \\
\text { 14. Materiais (ex. } \\
\text { brinquechila ou bolsa de bebê, comida, } \\
\text { cadeira de rodas). } \\
\text { 15. Informaçãa (ex. receber orientação sobre como realizar } \\
\text { as atividades, sobre eventos, serviços e } \\
\text { recursos disponíveis). }\end{array}$ \\
\hline
\end{tabular}

* Os termos tachados foram excluídos dos itens

** Os termos sublinhados se referem aos termos adicionados.

*** Modificações realizadas também nos ambientes da escola e da comunidade.

Na etapa 4 - entrevistas cognitivas com pais/ responsáveis - quatro pesquisadores analisaram os relatos coletados e procederam às seguintes modificações: alguns termos foram sublinhados nas instruções, foram feitas algumas substituições de itens por outros de melhor compreensão e foram incluídos exemplos nas questões. Nesta etapa, os maiores índices de dificuldade por parte dos respondentes se referiam aos itens da seção ambiente (aspectos físicos, mentais e sociais exigidos para realizar as atividades) julgados como pouco compreensíveis, assim como outros itens em que se entendia que algumas crianças eram muito pequenas para realizá-los. Ao finalizar as entrevistas, foi decidido pelos pesquisadores elaborar um material complementar de instruções para autoaplicação da ferramenta. Os dados sóciodemográficos dos entrevistados são apresentados na Tabela 2 e os resultados desta etapa, na Tabela 3.

Tabela 2 - Dados sócio-demográficos das participantes das entrevistas cognitivas

\begin{tabular}{|c|c|c|c|c|c|c|c|}
\hline Part. & Cidade -Estado & $\begin{array}{l}\text { Parentesco e } \\
\text { idade }\end{array}$ & Escolaridade & Ocupação & Renda familiar & $\begin{array}{l}\text { Sexo e idade } \\
\text { da criança }\end{array}$ & $\begin{array}{l}\text { Diagnóstico } \\
\text { Clínico }\end{array}$ \\
\hline 01 & Guarabira, PB & Mãe, 39 anos & Especialista & Contadora & Mais que $5 \mathrm{SM}$ & F - 3,11 anos & - \\
\hline 02 & Guarabira, PB & Mãe, 31 anos & Especialista & $\begin{array}{l}\text { Engenheira } \\
\text { Civil }\end{array}$ & Mais que $5 \mathrm{SM}$ & $\mathrm{M}-2,11$ anos & - \\
\hline 03 & Guarabira, PB & Mãe, 30 anos & Doutorado & Farmacêutica & Mais que $5 \mathrm{SM}$ & F - 3,6 anos & - \\
\hline 04 & Guarabira, PB & Mãe, 40 anos & Especialista & Psicóloga & Mais que $5 \mathrm{SM}$ & $\mathrm{M}-4,10$ anos & - \\
\hline 05 & Guarabira, PB & Mãe, 24 anos & Especialista & Enfermeira & 2 a $3 \mathrm{SM}$ & $\mathrm{M}-2,10$ anos & - \\
\hline
\end{tabular}


Silva Filho JA, et al. Medida da Participação e do Ambiente - Crianças Pequenas. Rev Ter Ocup Univ São Paulo. 2020 set./dez.;30(3):140-8.

Tabela 2 - Dados sócio-demográficos das participantes das entrevistas cognitivas

\begin{tabular}{|c|c|c|c|c|c|c|c|}
\hline Part. & Cidade -Estado & $\begin{array}{l}\text { Parentesco e } \\
\text { idade }\end{array}$ & Escolaridade & Ocupação & Renda familiar & $\begin{array}{l}\text { Sexo e idade } \\
\text { da criança }\end{array}$ & $\begin{array}{l}\text { Diagnóstico } \\
\text { Clínico }\end{array}$ \\
\hline 06 & Macaíba, RN & Mãe, 31 anos & $\begin{array}{l}\text { Fundamental } \\
\text { incompleto }\end{array}$ & Confeiteira & $1 \mathrm{SM}$ & $\mathrm{F}-4,9$ anos & Microcefalia \\
\hline 07 & Macaíba, RN & Mãe, 40 anos & Ensino médio & Agricultora & $1 \mathrm{SM}$ & M - 3,6 anos & Hidrocefalia \\
\hline 08 & Macaíba, RN & Mãe, 24 anos & $\begin{array}{l}\text { Ensino médio } \\
\text { incompleto }\end{array}$ & Dona de casa & $1 \mathrm{SM}$ & $\mathrm{F}-1,3$ anos & Paralisia cerebral \\
\hline 09 & Lagoa salgada, RN & Mãe, 24 anos & $\begin{array}{l}\text { Ensino } \\
\text { Fundamental }\end{array}$ & Dona de casa & $1 \mathrm{SM}$ & $\mathrm{M}-2,5$ anos & $\begin{array}{l}\text { Hidrocefalia } \\
\text { Mielomeningocele }\end{array}$ \\
\hline 10 & São Gonçalo, RN & Mãe, 29 anos & Ensino médio & Dona de casa & $1 \mathrm{SM}$ & F - 3,7 anos & $\begin{array}{l}\text { Microcefalia } \\
\text { Paralisia cerebral }\end{array}$ \\
\hline 11 & São Carlos, SP & Avó, 51 anos & Ensino médio & Cabeleireira & 4 a $5 \mathrm{SM}$ & $\mathrm{M}-1,7$ anos & $\begin{array}{l}\text { Má formação } \\
\text { cardíaca }\end{array}$ \\
\hline 12 & São Carlos, SP & Mãe, 40 anos & Ensino médio & Dona de casa & 4 a $5 \mathrm{SM}$ & $\mathrm{F}-10$ meses & $\begin{array}{l}\text { Síndrome de } \\
\text { Down }\end{array}$ \\
\hline 13 & São Carlos, SP & Mãe, 34 anos & Mestrado & $\begin{array}{l}\text { Assistente } \\
\text { Social }\end{array}$ & Mais que $5 \mathrm{SM}$ & $\mathrm{F}-1,3$ anos & - \\
\hline
\end{tabular}

Tabela 3 - Alterações dos itens na etapa 4 (Entrevistas cognitivas com pais ou responsáveis) da tradução e adaptação transcultural da YC-PEM

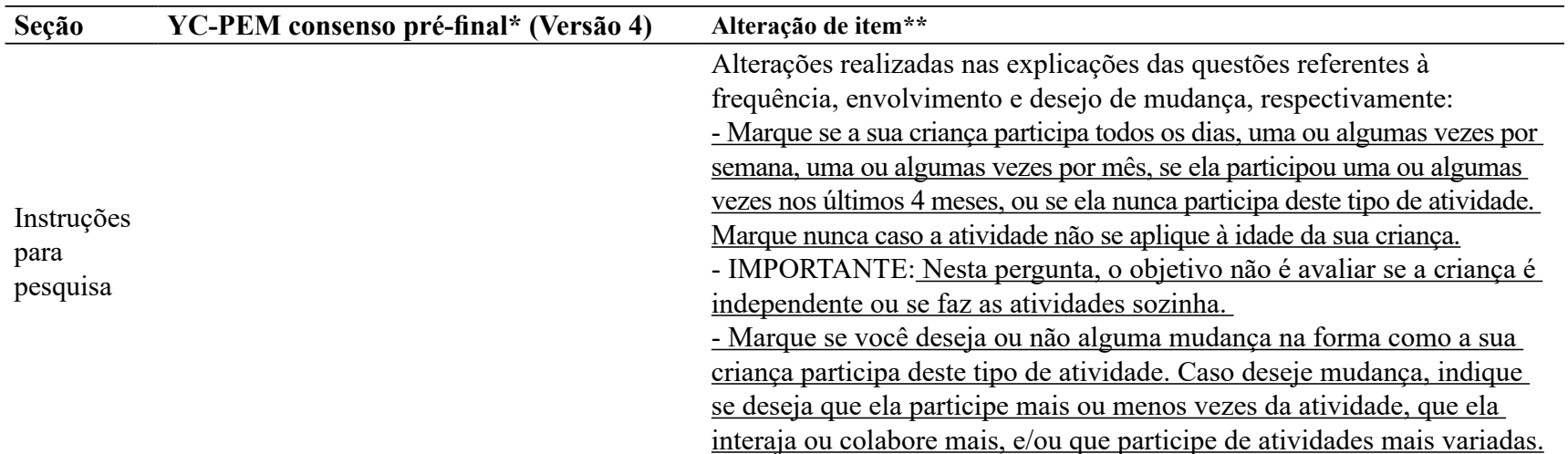

B1. timpeza (ex. guardar os brinquedos, usar a lixeira, limpar a mesa, arrumar a cama, Participação organizar suas coisas).

em casa B4. Lavar roupa e louça (ex. colocar, retirar, separar, dobrar ou guardar).

D1. Comemorações em casa (ex. festas de fim de ano, festas de-aniversário).

3. Os aspeetos físieos exigidos para realizar as atividades de casa (ex. força muscular, coordenação de movimentos, cansaço). 4. Os aspectos mentais exigidos para realizar Ambiente da as atividades de casa (ex. concentração, casa atenção, resolução de problemas).

5. Os aspetos sociais exigidos para realizar atividades de casa (ex. comunicação, interação com os outros).

$10-13 . \mathrm{N} / \mathrm{A}$.
B1. Organizacão (ex. guardar os brinquedos, usar a lixeira, limpar a mesa, arrumar a cama, organizar suas coisas).

B4. Lavar roupa ou louça (ex. colocar, retirar, separar, dobrar ou guardar).

D1. Comemorações em casa (ex. reuniões familiares, festas de fim de ano, aniversário).

3. As capacidades físicas necessárias para realizar atividades de casa (ex. força muscular, coordenação motora, cansaço).***

4. As capacidades mentais necessárias para realizar atividades de casa (ex. concentração, atenção, resolução de problemas).***

5. As capacidades sociais necessárias para realizar atividades de casa (ex. comunicação, interação com os outros).***

$10-13$. Não se aplica. ${ }^{* * *}$ 
Silva Filho JA, et al. Medida da Participação e do Ambiente - Crianças Pequenas. Rev Ter Ocup Univ São Paulo. 2020 set./dez:;30(3):140-9.

Tabela 3 - Alterações dos itens na etapa 4 (Entrevistas cognitivas com pais ou responsáveis) da tradução e adaptação transcultural da YC-PEM

\begin{tabular}{lll} 
Seção & YC-PEM consenso pré-final* (Versão 4) & Alteração de item** \\
\hline $\begin{array}{l}\text { Participação } \\
\text { na creche/ } \\
\text { pré-escola }\end{array}$ & $\begin{array}{l}\text { A1. Atividades em grupo (ex. hora da roda, } \\
\text { hora da história, músiea, aulas de artes). }\end{array}$ & $\begin{array}{l}\text { A1. Atividades em grupo (ex. hora da roda, hora da história, aulas de } \\
\text { artes, esportes). }\end{array}$ \\
\hline $\begin{array}{l}\text { Ambiente da } \\
\text { creche/pré- } \\
\text { escola }\end{array}$ & $\begin{array}{l}\text { 10. Acesso a transporte pessoal para ir para a a } \\
\text { creche/pré-escola (ex. carro ou bicicleta). }\end{array}$ & $\begin{array}{l}\text { 10. Acesso a transporte pessoal para ir para a creche/pré-escola (ex. carro, } \\
\text { bicicleta ou moto). }\end{array}$ \\
\hline
\end{tabular}

\begin{tabular}{|c|c|c|}
\hline $\begin{array}{l}\text { Participação } \\
\text { na } \\
\text { comunidade }\end{array}$ & $\begin{array}{l}\text { A. Passeios no bairro e comunidade: } \\
\text { Neste tipo de atividade, a criança vai com } \\
\text { membros de sua família a espaços do bairro, } \\
\text { comunidade ou cidade, para adquirir bens } \\
\text { e objetos ou fazer coisas necessárias para } \\
\text { o cuidado pessoal, para a saúde ou para o } \\
\text { cuidado com a casa. }\end{array}$ & $\begin{array}{l}\text { A. Passeios } \\
\text { vai com me } \\
\text { cidade, parc } \\
\text { cuidado pes }\end{array}$ \\
\hline $\begin{array}{l}\text { Ambiente da } \\
\text { comunidade }\end{array}$ & $\begin{array}{l}\text { 11. Acesso a transporte pessoal para } \\
\text { participar de atividades na comunidade } \\
\text { (ex. carro ou bicicleta). }\end{array}$ & $\begin{array}{l}\text { 11. Acesso } \\
\text { comunidad }\end{array}$ \\
\hline
\end{tabular}

Na etapa 5 - retro tradução, a versão 5 (consenso final) originada do estágio precedente foi traduzida novamente para o inglês, idioma original do Instrumento, sendo este resultado (versão 6) enviado para o CanChild para sua avaliação (Etapa 6).

\section{DISCUSSÃO}

A tradução e adaptação transcultural de medidas são utilizadas por pesquisadores de diversas áreas de interesse, pois em comparação com a criação de novas ferramentas, trazem benefícios como economia de custos e de tempo, além de possibilitar a comparação entre realidades diferentes ${ }^{19}$. Para que um instrumento possa ser traduzido e adaptado satisfatoriamente, é exigida a fidelidade às técnicas e procedimentos padronizados ${ }^{20}$.

O processo ocorreu sem dificuldades consideráveis, respeitando-se a metodologia adotada e a proposta do instrumento. Todas as alterações e acréscimos de termos realizados nas instruções da ferramenta, bem como nos seus itens e opções de respostas, foram minuciosamente analisados e discutidos. Foram selecionados aqueles que melhor representassem a realidade sociocultural brasileira, mantivessem o significado semântico da versão original e, ao mesmo tempo, facilitassem a compreensão do público-alvo.

Durante o processo, notou-se que a idade da criança por vezes parecia ser um fator dificultador para responder a alguns itens, cujas atividades eram consideradas pelos pais/ responsáveis respondentes como inadequadas para crianças muito pequenas. $\mathrm{O}$ nível educacional destes respondentes também teve influência no envolvimento com a pesquisa; os que tinham maior nível de escolarização foram mais críticos e sugeriram adaptações, contrastando com outros que precisavam, por vezes, de explicação sobre os itens e opções de resposta, o que fomentou a formulação de um material complementar de instruções, também necessário na adaptação brasileira do PEM-CY ${ }^{16}$.

As propriedades psicométricas da YC-PEM foram avaliadas na América do Norte com 395 crianças (sendo 93 com incapacidades). Os resultados obtidos dão suporte inicial ao uso da YC-PEM em pesquisas que almejam avaliar a participação de crianças pequenas com deficiência e atraso de desenvolvimento em termos de (1) padrões de participação em casa, creche/pré-escola e comunidade; (2) facilitadores e barreiras ambientais para participação, e (3) estratégias parentais específicas para promover a participação ${ }^{13}$.

Considerando de fundamental importância o estabelecimento de uma equivalência cultural de conteúdo da YC-PEM, a versão brasileira foi elaborada buscando a maior proximidade com a realidade sociocultural do Brasil. A YCPEM também foi adaptada para utilização na Suécia $^{14} \mathrm{e}$ em Singapura, onde, apesar de usar o mesmo idioma, a maioria dos itens precisou sofrer modificação para ter aplicabilidade relevante para a cultura alvo ${ }^{21}$. A adaptação transcultural da YC-PEM é importante já que existem diferenças culturais 
na participação das crianças, seus ambientes e na forma como as pessoas entendem as palavras ${ }^{21}$.

AYC-PEM ainda não havia sido traduzida ao português e adaptada ao Brasil. Por ser originalmente autoaplicada, pode haver interferência em caso de dificuldades de leitura e interpretação dos itens por respondentes de regiões específicas do Brasil. De fato, alguns pais precisaram de ajuda para o preenchimento durante a etapa 4 desta pesquisa, o que motivou a sugestão da possibilidade de aplicação em formato de entrevista ou aplicada com ajuda, como também aconteceu na adaptação brasileira da PEM-CY ${ }^{16}$.

O Brasil é um país muito extenso e com distintas características culturais, assim, esta é uma pesquisa que deverá ter continuidade em outros estados, com o objetivo de avaliar as propriedades psicométricas do instrumento e consequentemente permitir sua validação para o uso no Brasil. Isso a tornará uma medida consistente e confiável para ser aplicada em nível nacional, além de servir de incentivo para outras pesquisas semelhantes envolvendo a participação de crianças e o ambiente no qual convivem.

\section{CONCLUSÃO}

A YC-PEM foi traduzida ao português do Brasil e adaptada transculturalmente para este país e o produto final, a YC-PEM Brasil (Medida da Participação e do Ambiente - Crianças Pequenas), foi considerado satisfatório. Tal resultado reflete o seguimento rigoroso de etapas de acordo com o referencial metodológico adotado. Esperase que a YC-PEM Brasil possa ajudar pais, responsáveis e profissionais da reabilitação a identificarem os níveis de participação e as características do ambiente de crianças entre zero e cinco anos de idade, permitindo assim o planejamento e a implementação de intervenções em reabilitação centradas nestes fatores de grande relevância para as crianças brasileiras.

Agradecimentos: Gostaríamos de agradecer a todas as crianças e suas responsáveis que participaram deste projeto. Nossa gratidão também às profissionais que constituíram o comitê de especialistas na etapa 3 e aos centros envolvidos no recrutamento das crianças com deficiência, como o Centro de Educação e Pesquisa em Saúde Anita Garibaldi (CEPS), localizado na cidade de Macaíba - RN e Unidade Saúde Escola (USE) da Universidade Federal de São Carlos, São Carlos - SP. Agradecemos ainda às Instituições de Ensino Superior que tornaram possível a realização desta pesquisa: Universidade Federal do Rio Grande do Norte - FACISA, Universidade Federal do Rio de Janeiro, Universidade Federal do Ceará e Universidade Federal de São Carlos.

Participação dos autores: Todos os autores participaram da concepção e desenho do estudo. Silva Filho JA, Campos AC: realizaram as coletas de dados. Silva Filho JA: escreveu o artigo. Cazeiro APM, Longo E: participaram do processo de tradução inicial. Campos AC: realizou a retro tradução. Silva Filho JA, Longo E, Campos AC, Cazeiro APM: participaram do processo de adaptação transcultural do instrumento. Longo E, Campos AC, Cazeiro APM: revisaram o manuscrito e forneceram feedback substancial na redação do artigo. Silva Filho JA: editou a versão final.

\section{REFERENCIAS}

1. Organização Mundial De Saúde - OMS. Classificação internacional de funcionalidade, incapacidade e saúde (CIF). Portugal: Lisboa; 2004.

2. Majnemer A. Promoting participation in leisure activities: expanding role for pediatric therapists. Phys Occup Ther Pediatr. 2009;29(1):1-5. doi: 10.1080/01942630802625163.

3. Law M. Participation in the occupations of everyday life. Am J Occup Ther. 2002;56(6):640-9. doi: 10.5014/ajot.56.6.640.

4. Coster W, Law M, Bedell G, Khetani M, Cousins M, Teplicky $\mathrm{R}$. Development of the participation and environment measure for children and youth: conceptual basis. Disabil Rehabil. 2012;34(3):238-46. doi: 10.3109/09638288.2011.603017.

5. Albrecht EC, Khetani MA. Environmental impact on young children's participation in home based activities. Dev Med Child Neurol. 2017;59(4):388-94. doi: 10.1111/dmcn.13360.

6. Law M, King G, King S, Kertoy M, Hurley P, Rosenbaum P. Patterns of participation in recreational and leisure activities among children with complex physical disabilities. Dev Med Child Neurol. 2006;48(5):337-42. doi: 10.1017/ S0012162206000740.

7. Badia M, Longo E, Orgaz BM, Gómez-Vela M. The influence of participation in leisure activities on quality of life in Spanish 
children and adolescents with cerebral palsy. Res Dev Disabil. 2013;34:2864-71. doi: 10.1016/j.ridd.2013.06.017.

8. Badia M, Orgaz BM, Gómez-Vela M, Longo E. Environmental needs and facilitators available for children and adolescents with cerebral palsy: adaptation and validation of the European Child Environment Questionnaire (ECEQ) Spanish version. Disabil Rehabil. 2014:1-13. doi: 10.3109/09638288.2013.847124.

9. Longo E, Badia M, Orgaz M. Patterns and predictors of participation in leisure activities outside of school in children and adolescents with cerebral palsy. Res Dev Disabil. 2013;34(1):266-75. doi: 10.1016/j.ridd.2012.08.017.

10. Law MC, Darrah J, Pollock N, Wilson B, Russel DJ, Walter $\mathrm{SD}$, et al. Focus on function: a cluster, randomized controlled trial comparing child- versus context-focused intervention for young children with cerebral palsy. Dev Med Child Neurol. 2011;53:621-9. doi: 10.1111/j.1469-8749.2011.03962.

11. Amaral M, Paula RL, Drummond A, Dunn L, Mancini MC. Translation of the Children Helping Out - Responsibilities, Expectations and Supports (CHORES) questionnaire into Brazilian Portuguese: semantic, idiomatic, conceptual and experiential equivalences and application in normal children and adolescents and in children with cerebral palsy. Rev Bras Fisioter. 2012;16(6):512-22. doi: 10.1590/S1413-35552012000600011.

12. Furtado SRC, Sampaio RF, Vaz DV, Pinho BAS, Nascimento IO, Mancini MC. Versão brasileira do instrumento de avaliação ambiental Craig Hospital Inventory of Environmental Factors (CHIEF): tradução, adaptação cultural e confiabilidade. Braz J Phys Ther. 2014;18(3). doi: 10.1590/bjpt-rbf.2014.0036.

13. Khetani MA, Graham JE, Davies PL, Law MC, Simeonsson RJ. psychometric properties of the young children's participation and environment measure. Arch Phys Med Rehabil. 2014;96(2):307-16. doi: 10.1016/j.apmr.2014.09.031.

14. Astrom FM, Khetani M, Axelsson AK. Young children's participation and environment measure: Swedish cultural adaptation. Phys Occup Ther Pediatr. 2017;1(14):1-15. doi: 10.1080/01942638.2017.1318430.

15. Jeong Y, Law M, Stratford P, Dematteo C, Kim H. Measuring Participation of Children and Environmental Factors at Home, School, and in Community: Construct Validation of the Korean PEM-CY. Phys Occup Ther Pediatr. 2017;7:1-13. doi: 10.1080/01942638.2017.1280870.

16. Galvão ERVP, Cazeiro APM, de Campos AC, Longo E. Medida de Participação e do Ambiente - Crianças e Jovens (PEM-CY): adaptação transcultural para o uso no Brasil. Rev Ter Ocup Univ São Paulo. 2018;29(3):237-45. doi: 10.11606/ issn.2238-6149.v29i3p237-245.

17. Beaton DE, Bombardier C, Guillemin F, Ferraz MB. Guidelines for the process of cross-cultural adaptation of self-report measure. Spine. 2000;25(4):3186-91. doi: 10.1097/00007632-200012150-00014.

18. Paulo RM, Albuquerque PB, Bull R. A entrevista cognitiva melhorada: pressupostos teóricos, investigação e aplicação. Rev Psicol. 2014;28(2):21-30.

19. Arthur JP, Mantovani MF, Ferraz MIR, Mattei AT, Kalinke LP, Corpolato RC. Tradução e Adaptação Transcultural da Hipertension Knowledge-Level Scale para uso no Brasil. Rev Latino-Am Enfermagem. 2018;16(e3073). doi: 10.1590/1518-8345.2832.3073.

20. Coster W, Bedell J, Law M, Khetani MA, Teplicky R, Liljenquist K, Gleason K, Kao Y. Psychometric evaluation of the Participation an Environment Measure for Children and Youth (PEM-CY). Dev Med Child Neurol. 2011;53(11):10307. doi: 10.1111/j.1469-8749.2011.04094.x.

21. Lim CY, Law M, Khetani M, Pollock N, Rosenbaum P. Establishing the Cultural Equivalence of the Young Children's Participation and Environment Measure (YC-PEM) for Use in Singapore. Phys Occup Ther Pediatr. 2016;36 (4):422-39. doi: 10.3109/01942638.2015.1101044. 